\section{Financiamento público em saúde e confiabilidade dos bancos de dados nacionais. Um estudo dos anos de 2001 e 2002}

\author{
Government funding for health and the reliability \\ of national databases in Brazil, 2001-2002
}

\author{
1 Secretaria Executiva, \\ Ministério da Saúde, \\ Rio de Janeiro, Brasil. \\ 2 Escola Nacional de Saúde \\ Pública Sergio Arouca, \\ Fundação Oswaldo Cruz, \\ Rio de Janeiro, Brasil. \\ Correspondência \\ C. R. A. Lima \\ Secretaria Executiva, \\ Ministério da Saúde. \\ Rua México 128, 8o andar, \\ Rio de Janeiro, $R J$ \\ 20031-142, Brasil. \\ crisso@datasus.gov.br
}

\begin{abstract}
Created in 1999, the Information System on Government Health Budgets (SIOPS) provides information on health revenues and expenditures at the three government levels: Municipal, State, and Federal. The lack of other databases with nationwide coverage and detailed information on municipal expenditures makes SIOPS the main source of data for such studies or estimates. The current study aims to compare the revenues declared in SIOPS by the municipalities and the National Health Fund records in order to assess the reliability of the two databases and identify which variables are most discordant. It also compares the data on Municipal expenditures with those from National Treasury records. The Intra-Class Correlation Coefficient (CCIC) was used for this analysis, complemented by a scatterplot of the mean values for the two databases and their differences. The 2002 data showed better quality. The recognition that SIOPS provides reliable data should stimulate new studies including financial aspects in the analysis of population health.
\end{abstract}

Health Financing; Information Systems; Health Systems
Claudia Risso de Araujo Lima 1

Marilia Sá Carvalho ${ }^{2}$

Joyce Mendes de Andrade Schramm 2

\section{Introdução}

No Brasil, no ano 2000, foram gastos R\$ 34 bilhões em ações e serviços de saúde pública, correspondendo a $\mathrm{R} \$ 201,00$ por habitante-ano e a $3,09 \%$ do produto interno bruto (PIB). Estimase que, com a promulgação da Emenda Constitucional $n .291$, que assegura a efetiva co-participação da União, dos Estados, do Distrito Federal e dos Municípios no financiamento das ações e serviços públicos de saúde, as despesas conjuntas das três esferas de governo tenham aumentado mais de $30 \%$ entre 2000 e 2004 2,3,4,5.

Acompanhar as transferências e o comportamento das instâncias subnacionais em relação aos gastos públicos é essencial para a análise do processo de descentralização do SUS. Uma fonte de informações sobre as transferências voluntárias para níveis subnacionais de governo e para instituições privadas é o Sistema de Acompanhamento Financeiro (Siafi) e o Sistema Integrado de Dados Orçamentários (Sidor) do governo federal. Contudo, a regionalização dos dispêndios federais com saúde baseando-se no Siafi/Sidor não é completa, uma vez que boa parte dos projetos/atividades no campo da saúde não apresenta a destinação geográfica inequívoca 6 . As informações sobre os dispêndios com pagamentos a provedores e os repasses para estados e municípios feitos pelo Fundo Nacional de Saúde (FNS) estão disponíveis na página eletrônica do Departamento 
de Informação e Informática do SUS (DATASUS) 7 e do FNS 8.

Entretanto, obter informações financeiras detalhadas de base municipal é uma tarefa mais difícil. A Fundação Getúlio Vargas disponibilizava, até 1998, informações sobre gasto público de uma amostra de 186 municípios, que incluía as capitais e os municípios das regiões metropolitanas. A última consolidação disponível era relativa a dez anos antes 9. Desde 1989 o sistema Finanças do Brasil (FINBRA) 10, da Secretaria do Tesouro Nacional, disponibiliza informações sobre receitas municipais e despesas com saúde e saneamento, entretanto os dados relativos a despesas estão agrupados.

Com o objetivo de coletar informações sobre receita e despesa com ações e serviços de saúde das três esferas de governo, em 1999 foi criado o Sistema de Informações sobre Orçamentos Públicos em Saúde (SIOPS) 11. A inexistência de outros bancos de dados de abrangência nacional com informações detalhadas sobre os gastos municipais faz com que o SIOPS seja a principal fonte de dados para estudos ou estimativas desta natureza.

Este trabalho compara os dados do SIOPS sobre transferências da União para a área de saúde dos municípios com os registros do FNS, no intuito de verificar a confiabilidade dos dois bancos de dados e identificar as variáveis com maior diferença. Compara também os valores declarados ao SIOPS para despesas totais com saúde com os registros disponíveis na Secretaria do Tesouro Nacional (STN).

\section{Método}

Este estudo foi realizado com os dados de receita arrecadada e despesa realizada (liquidada), da administração direta e indireta, dos municípios que enviaram dados ao SIOPS até 31 de outubro de 2003 relativos ao ano-base 2001 (4.908 municípios) e ao ano-base 2002 (4.695 municípios). Estes dados foram comparados com os registros do banco do FNS de transferências realizadas para o Fundo Municipal de Saúde desses municípios e com os registros de despesa com saúde do FINBRA. Decidiu-se trabalhar com os dados a partir do ano de 2001 devido à incorporação de um maior número de críticas ao programa de entrada de dados do SIOPS nesse ano e à disponibilidade dos dados do FNS.

As transferências regulares do FNS para o Fundo Municipal de Saúde estão discriminadas no SIOPS em: (a) Piso de Atenção Básica fixo (PAB fixo); (b) Piso de Atenção Básica variá- vel (PAB variável); (c) Transferência de Alta e Média Complexidade (MAC) - Sistema de Informação Ambulatorial/Sistema de Informação Hospitalar (SIA/SIH); (d) Transferências do Fundo de Ações Estratégicas e Compensação (FAEC); (e) Convênios da área de saúde. Para o ano de 2001 apenas o PAB variável está detalhado no SIOPS, enquanto que no banco de dados do FNS também os dados relativos a MAC e FAEC estão desagregados por cada programa ou campanha do Ministério da Saúde (MS), procedimento adotado pelo SIOPS no ano seguinte. Para possibilitar a comparação entre esses dois bancos de dados para o ano de 2001, os itens do FNS foram agregados conforme definição de sua composição obtida em reunião com a equipe responsável pelo sistema.

Foram selecionadas para o presente estudo as variáveis com pelo menos $80 \%$ dos municípios com dados informados simultaneamente nos dois bancos de dados, dentre o total de municípios com informações para a variável. Além destas, foram incluídos os valores transferidos para MAC por representarem para o ano de 2001 pouco mais de $50 \%$ do montante transferido aos municípios.

O Coeficiente de Correlação Intraclasses (CCIC), que tem sido o método mais utilizado para verificar consistência dos dados para valores contínuos, principalmente quando não se pode garantir com exatidão que um dos bancos de dados seja o padrão ouro, foi escolhido para a comparação entre as fontes de informação. A correlação é obtida a partir da combinação da medida de correlação com teste de diferenciação de médias. Verifica a similaridade de interceptos, portanto, se uma variável é sistematicamente maior, o $r$ obtido é reduzido. Os valores podem variar de -1 a 1 . No entanto, este método não é suficiente para verificar concordância entre valores, pois duas variáveis podem estar estreitamente relacionadas, apresentando o mesmo comportamento, mas nunca concordarem os valores. Estudos realizados apresentam, como complemento ao CCIC, o gráfico entre a média dos valores dos dois bancos de dados e a diferença entre estes valores. O método gráfico permite a visualização do viés e dos padrões distintos de confiabilidade segundo diferentes estratos e níveis da variável mensurada. Para avaliar a magnitude da discordância entre os valores, pesos são atribuídos de acordo com a categoria obtida $12,13,14$.

A comparação dos dados de receita, em que um banco de dados contém informações fornecidas pelo órgão responsável por repassar os recursos financeiros e outro banco de dados 
contém os valores declarados pela instância que os recebe, não deveria apresentar grandes diferenças entre os valores registrados. Portanto, calculou-se o CCIC e o estudo foi complementado com gráficos de dispersão. Estes diagramas foram obtidos calculando-se a média e a diferença entre o valor encontrado no SIOPS e o valor registrado em cada banco utilizado para conferência: FNS (SIOPS - FNS) e FINBRA (SIOPS-FINBRA). Os valores extremos foram retirados da análise para uma melhor visualização do gráfico, entretanto, para nenhuma variável foram excluídos mais do que dez municípios.

Considerando o regime de contabilização de receitas e despesas vigente no Brasil, onde as receitas são apropriadas pelo regime de caixa e as despesas pelo de competência, foi atribuído peso 0 para diferenças inferiores a $10 \%$. Para diferença positiva entre 11 e $20 \%$ foi atribuído peso 1, -1 para diferença negativa nesta faixa, 2 para diferença positiva maior do que $20 \%$ e - 2 para diferença negativa nesta faixa. A análise destas diferenças foi realizada para diferentes estratos.

Todo o estudo foi desenvolvido utilizandose o aplicativo TabWin (http://www.datasus. gov.br/tabwin/tabwin.htm) e os esquemas de análise dos programas estatísticos R (http:/ / www. R-project.org), disponíveis no TabWin e SPSS (SPSS Inc., Chicago, Estados Unidos).

\section{Resultados}

Os achados demonstram que, para o ano de 2001, o FNS registrou uma maior quantidade de municípios com transferências para a área de saúde do que os registrados no SIOPS. Os itens de Atenção Básica apresentaram para esse ano um alto registro simultâneo nesses dois bancos: $98 \%$ dos municípios para PAB fixo e $90 \%$ para PAB variável. Já os itens que compõem o PAB variável, quando detalhados, apresentaram uma maior diferença entre os dois bancos de dados, principalmente para os recursos transferidos para Ações de Atenção à Saúde dos Povos Indígenas, Programa de Combate às Carências Nutricionais, Programa Nacional de Vigilância Epidemiológica e Controle de Doenças e Programa de Assistência Farmacêutica Básica.

Os recursos transferidos que não destinados são para atenção básica (média e alta complexidade - SIH/SIA, FAEC e Convênios) e apresentaram menos de $40 \%$ dos municípios com informações nos dois bancos simultaneamente. O SIOPS apresentou 53\% de municípios a mais que o FNS com informações sobre convênios firmados com o FNS.

A variável que apresentou o menor número de municípios com dados para o ano 2001 simultaneamente nesses dois bancos de dados foi MAC (35\% dos municípios), enquanto que para PAB fixo apenas $0,4 \%$ não apresentou informações nos dois bancos.

Em 2002, a ocorrência simultânea de informações nos dois bancos de dados é maior do que $90 \%$ para quase todas as variáveis, com exceção apenas para os programas de Ações de Atenção à Saúde dos Povos Indígenas e de Assistência Farmacêutica Básica.

As variáveis que atingiram, nos dois anos, pelo menos $80 \%$ de municípios com registros nesses dois bancos simultaneamente foram PAB fixo, PAB variável e seu detalhamento: Ações Básicas de Vigilância Sanitária, Programa Saúde da Família (PSF), Programa de Agentes Comunitários de Saúde (PACS) e total de transferências da União para a saúde, todas relativas à atenção básica (com exceção do total). Entretanto, MAC também foi selecionada para o estudo, incorporando assim uma variável não PAB com grande peso no montante das transferências.

A Tabela 1 permite verificar alta correlação (acima de 0,80 ) para quase todas as variáveis nos dois anos analisados, com exceção apenas para Ações Básicas de Vigilância Sanitária, que apresentou uma baixa correlação para o ano de 2001 (apenas 0,38 ) e PSF $(0,73)$ para o ano de 2002. Variações acentuadas entre os dois anos são percebidas apenas para Ações Básicas de Vigilância Sanitária (positiva) e PSF (negativa). Para o ano de 2002, as correlações encontradas para as variáveis PAB fixo, PAB variável, MAC, total das transferências e despesas totais com saúde estão bem próximas da unidade. A Tabela 1 demonstra que não houve uma grande variação na quantidade de municípios analisados em cada ano, apenas a variável PAB fixo apresentou um menor número de municípios com dados no segundo ano analisado.

Apesar das altas correlações encontradas, observa-se dispersão acentuada para o ano de 2001 para todas as variáveis, conforme pode ser observado na Figura 1. Para esse ano, os achados evidenciam uma relação entre a média (expressa no eixo x) e a diferença entre os valores dos bancos de dados (expressa no eixo y) para todas as variáveis da receita. Entretanto, verifica-se uma grande diferença no padrão apresentado para essas variáveis nos dois anos analisados, principalmente para PAB fixo, constatando-se em 2002 uma relação bastante discreta ou inexistente, evidenciada pela aproxi- 
Total de municípios analisados, Coeficiente de Correlação Intraclasses $(\mathrm{CCIC})$ e intervalo de confiança a 95\% (IC95\%) para os anos de 2001 e 2002 para as variáveis analisadas.

\begin{tabular}{|c|c|c|c|c|c|c|}
\hline \multirow[t]{2}{*}{ Variável } & \multicolumn{3}{|c|}{2001} & \multicolumn{3}{|c|}{2002} \\
\hline & $\mathrm{n}$ & $\mathrm{CCIC}$ & IC95\% & $n$ & $\mathrm{CClC}$ & IC95\% \\
\hline Piso de Atenção Básica fixo & 4.806 & 0,9313 & $0,9275-0,9350$ & 4.683 & 0,9767 & $0,9753-0,9779$ \\
\hline Piso de Atenção Básica variável & 4.373 & 0,8693 & $0,8619-0,8763$ & 4.688 & 0,9899 & $0,9893-0,9905$ \\
\hline Programa de Vigilância Sanitária & 3.883 & 0,3781 & $0,3508-0,4047$ & 4.459 & 0,8158 & $0,8057-0,8254$ \\
\hline Programa Saúde da Família & 2.809 & 0,9646 & $0,9619-0,9671$ & 3.600 & 0,7349 & $0,7195-0,7495$ \\
\hline $\begin{array}{l}\text { Programa de Agentes } \\
\text { Comunitários de Saúde }\end{array}$ & 3.513 & 0,9652 & $0,9628-0,9674$ & 4.053 & 0,9736 & $0,9720-0,9752$ \\
\hline Média e Alta Complexidade & 460 & 0,9866 & $0,9840-0,9888$ & 517 & 0,9939 & $0,9927-0,9949$ \\
\hline Total de Transferências & 4.889 & 0,9874 & $0,9866-0,9881$ & 4.695 & 0,9916 & $0,9911-0,9921$ \\
\hline Despesa Total com Saúde & 4.749 & 0,9750 & $0,9735-0,9763$ & 4.068 & 0,9851 & $0,9842-0,9860$ \\
\hline
\end{tabular}

Fonte: Sistema de Informações sobre Orçamentos Públicos em Saúde, Ministério da Saúde/

Fundo Nacional de Saúde/sistema Finanças do Brasil, Secretaria do Tesouro Nacional.

mação das linhas de tendência ao eixo do x no segundo ano. Os valores referentes para a despesa (comparação entre o SIOPS e FINBRA) mantiveram um padrão mais constante entre os anos de 2001 e 2002, conforme pode ser observado na Figura 2.

A Figura 3 apresenta a magnitude dessas diferenças. Os achados demonstram, para o ano de 2001 , diferenças inferiores a $10 \%$ em mais de $70 \%$ dos municípios que informaram Ações Básicas de Vigilância Sanitária (PVS) e MAC, mas superiores a $20 \%$ para mais da metade dos municípios que informaram PAB fixo e PAB variável. As diferenças encontradas entre o SIOPS e o FINBRA (despesas) são inferiores a 10\% dos valores informados ao SIOPS para $51 \%$ dos municípios, entre 11 e $20 \%$ para quase $19 \%$ e superiores a $20 \%$ para $30 \%$ dos municípios.

Para o ano de 2002, como já demonstrado nos gráficos de dispersão, as diferenças são bem menores, com pelo menos $75 \%$ dos municípios apresentando diferenças inferiores a $10 \%$ para quase todas as variáveis, com exceção apenas para PACS, onde $47 \%$ dos municípios ainda informam ao SIOPS valores entre 11 e $20 \%$ superiores aos registrados pelo FNS. As diferenças entre os dados de despesa se apresentaram ainda menores do que no ano anterior, com apenas $22 \%$ dos municípios com diferenças superiores a $10 \%$, a metade destas superiores a $20 \%$. Para esse ano os achados não demonstram uma relação entre a diferença entre os valores do SIOPS e dos demais bancos de dados e o porte populacional.

Dentre os municípios selecionados para o ano de 2001, 4.503 municípios estavam em gestão plena da atenção básica, 368 em gestão ple- na do sistema municipal e 37 sem habilitação. Nesse ano, as maiores variações para os itens de PAB variável entre os bancos de dados ocorreram nos municípios em gestão plena de atenção básica. Para o PAB fixo, as duas condições de gestão apresentaram o mesmo comportamento. A partir do ano de 2002, 4.112 municípios se apresentaram em gestão plena da atenção básica municipal e 566 em gestão plena do sistema. Nesse ano, diferenças inferiores a $10 \%$ são percebidas em 94\% dos municípios em gestão plena da atenção básica e em $91 \%$ em gestão plena do sistema.

A diferença entre os sistemas para o total de transferências apresentou uma distribuição espacial quase uniforme no país nos dois anos, quando analisada por município. Mas a análise por região apresentou variações nos dois anos analisados, com as maiores diferenças ocorrendo nas regiões Centro-oeste e Norte e as menores no Nordeste $(1,31 \%)$ e Sul $(0,53 \%)$. Para o ano de 2001, a região Sudeste foi a única que apresentou os valores do FNS superiores (em $3,46 \%$ ) aos valores informados ao SIOPS em todos os seus estados. Entretanto, diferentemente dos dados da receita, as maiores diferenças dos dados de despesa estão localizadas nas regiões Nordeste e Norte, e as demais regiões se distribuíram bastante igualitariamente. Em 2002, as regiões Sul e Sudeste apresentaram dados no FNS superiores ao SIOPS, com a menor diferença no Sudeste. Para os dados de despesa as menores diferenças estavam localizadas nas regiões Sudeste e Sul e como no ano anterior, nas menores faixas de população.

As variáveis apresentaram um comportamento bastante distinto nos dois bancos de da- 
Diagrama de dispersão da diferença e a média dos valores entre Sistema de Informações sobre Orçamentos Públicos em Saúde e Fundo Nacional de Saúde, para os anos de 2001 e 2002 para cada uma das variáveis.
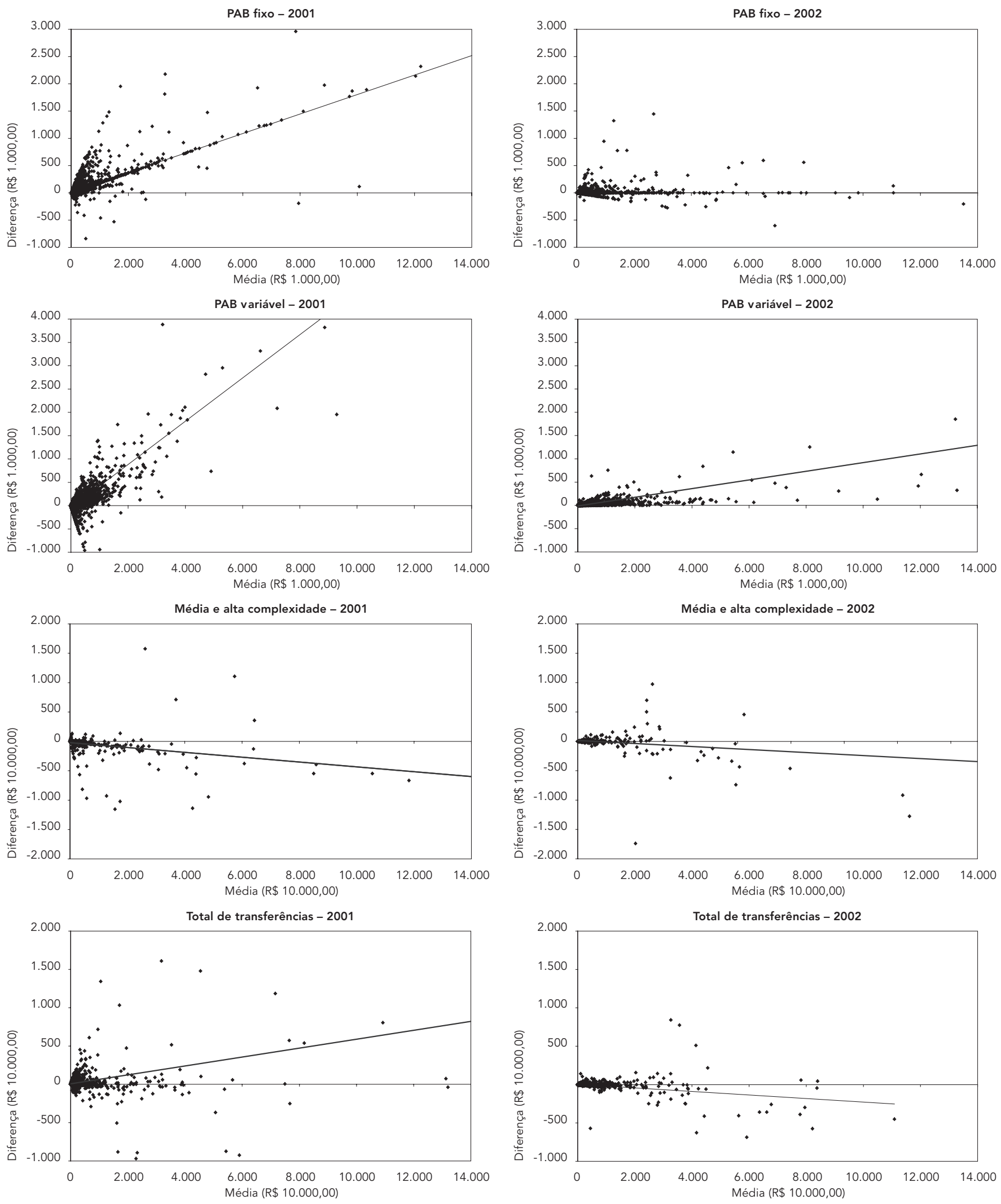
Diagrama de dispersão da diferença e a média dos valores entre Sistema de Informações sobre Orçamentos Públicos em Saúde e sistema Finanças do Brasil, para os anos de 2001 e 2002.
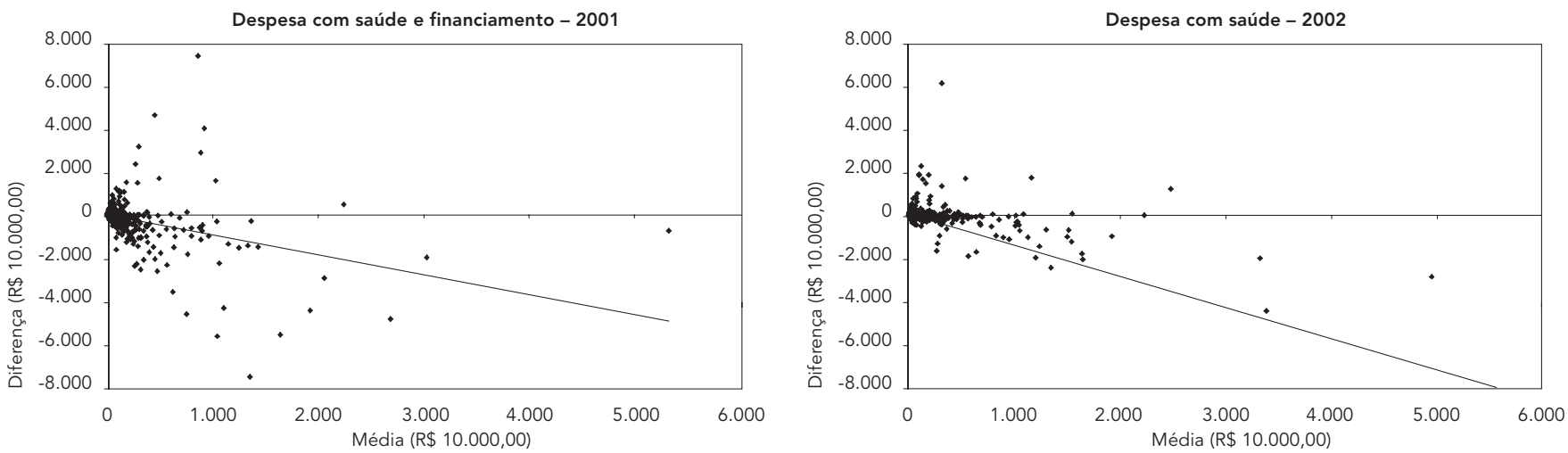

dos quando se analisa a variação entre os valores para cada ano, conforme demonstra a Figura 4. As maiores variações foram encontradas no sistema do FNS, onde os registros dos valores de PACS, e conseqüentemente do PAB variável, acresceram de um ano para o outro mais de $20 \%$ em cerca de $90 \%$ dos municípios analisados. As variáveis PAB fixo e PSF também apresentaram acréscimos nesta faixa para aproximadamente $60 \%$ dos municípios. Apenas MAC manteve um grande percentual de municípios com variação inferior a $10 \%$. Já no banco de dados do SIOPS, somente PACS, e conseqüentemente $\mathrm{PAB}$ variável, apresentaram grandes variações. As variações encontradas no FINBRA foram inferiores às encontradas nos dados de despesa do SIOPS.

A correlação (CCIC - para IC95\%) encontrada entre os dois anos para o SIOPS para os dados de receita foi de 0,9715 (0,9698-0,9731), para despesa foi de $0,9848(0,9838-0,9857)$, para o FNS foi de 0,9275 (0,9233-0,9315) e para o FINBRA foi de 0,9736 (0,9719-0,9752).

\section{Discussão}

A acentuada diferença observada entre o banco de dados do SIOPS e do FNS para os municípios que informaram recebimentos de dados para Convênios em 2001 pode ser explicada por falhas na codificação de municípios, já que os dados fornecidos pelo FNS não dispunham do código de identificação do Instituto Brasi- leiro de Geografia e Estatística (IBGE) e erros podem ter ocorrido na codificação manual. A menor ocorrência simultânea de informações, em 2002, nos bancos do SIOPS e do FNS para a Assistência Farmacêutica Básica pode ser explicada por registros, no FNS, para os estados, mesmo quando o valor tenha sido repassado para os fundos municipais. Em relação ao programa de Ações de Atenção à Saúde dos Povos Indígenas, a diferença encontrada pode ser resultante de dificuldades na organização dos municípios pequenos, que recebem esse incentivo. Apenas a verificação dos balancetes municipais pode comprovar estas hipóteses.

As variações entre os bancos encontradas para MAC foram bem menores que aquelas encontradas para os itens de atenção básica. Estes itens apareceram com maiores diferenças nos municípios em gestão plena da atenção básica, sugerindo que os municípios pequenos têm maior dificuldade em obter e organizar suas informações. Isto pode ser observado também nas maiores diferenças encontradas para $\mathrm{PAB}$ variável quando detalhado por programa, e para os valores registrados em MAC em municípios com condição de gestão plena da atenção básica, sugerindo que os pagamentos realizados diretamente aos prestadores de serviço foram registrados em transferências fundo a fundo. Alguns municípios pequenos contratam escritórios de contabilidade para auxiliá-los a informar os dados solicitados pelo sistema. O apoio que os núcleos estaduais do SIOPS vêm realizando junto aos responsáveis pelo preen- 
Diferenças percentuais entre Sistemas de Informações sobre Orçamentos Públicos em Saúde (SIOPS) e Fundo Nacional de Saúde (FNS) e entre SIOPS e sistema Finanças do Brasil (FINBRA), por item analisado, para os anos de 2001 e 2002.

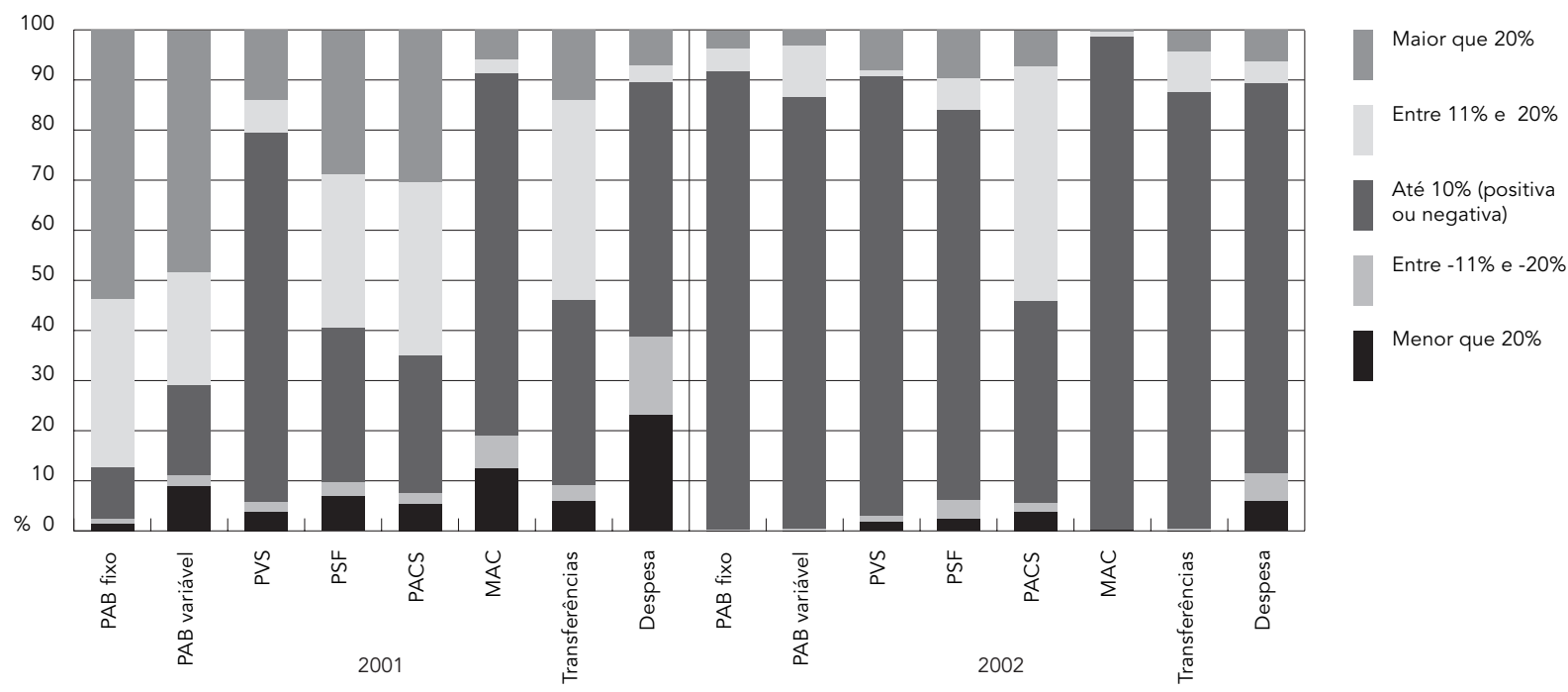

PAB fixo = Piso de Atenção Básica fixo; PAB variável = Piso de Atenção Básica variável; PVS = Ações Básicas de Vigilância Sanitária; PSF = Programa Saúde da Família; MAC = transferência de alta e média complexidade.

Figura 4

Diferenças porcentuais entre os anos de 2001 e 2002 para o banco de dados do Fundo Nacional de Saúde (FNS), sistema Finanças do Brasil (FINBRA) e Sistemas de Informações sobre Orçamentos Públicos em Saúde (SIOPS).

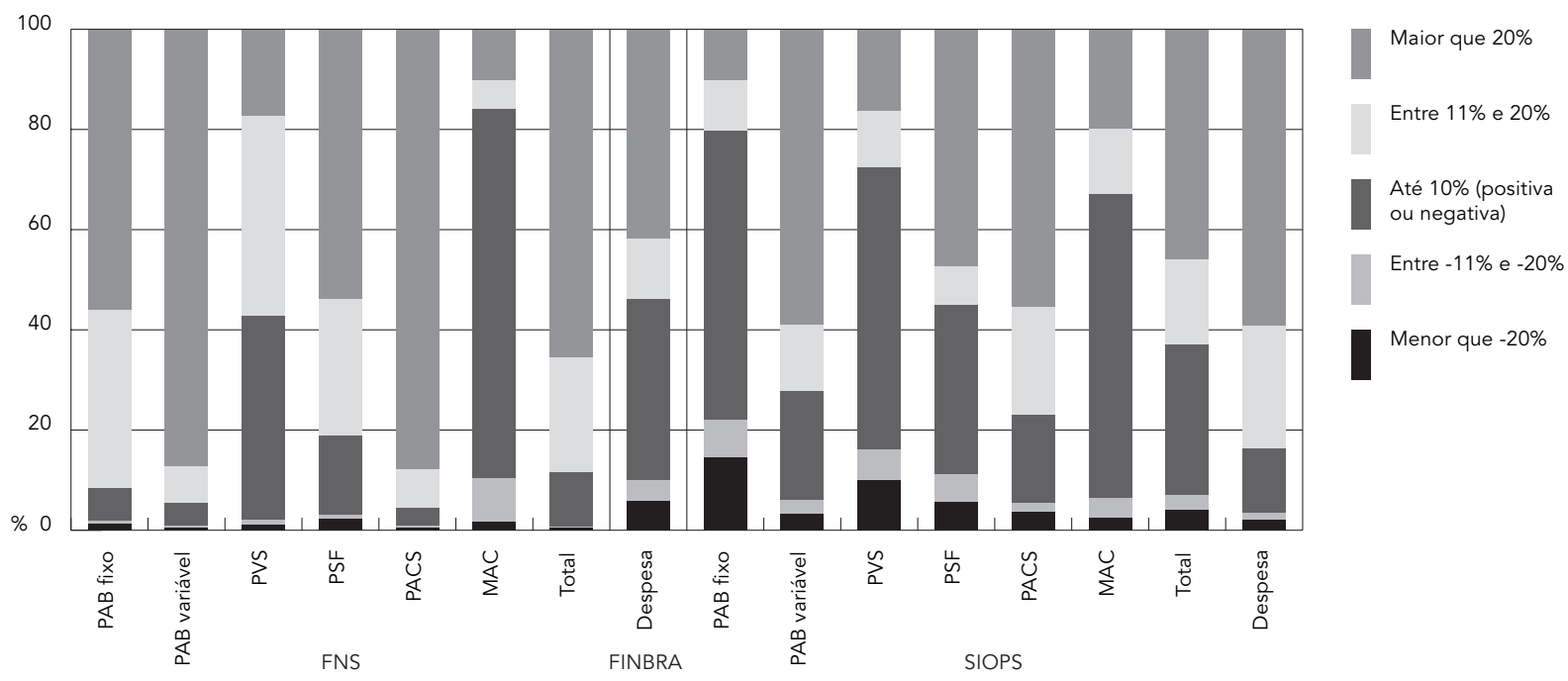

PAB fixo = Piso de Atenção Básica fixo; PAB variável = Piso de Atenção Básica variável; PVS = Ações Básicas de Vigilância Sanitária; PSF = Programa Saúde da Família; PACS = Programa de Agentes Comunitários de Saúde; $\mathrm{MAC}=$ transferência de alta e média complexidade. 
chimento das planilhas, por meio de ações de esclarecimento de dúvidas e capacitação no preenchimento das planilhas, deve resultar na melhoria da qualidade dos dados informados ao SIOPS.

Entretanto os gráficos de dispersão evidenciam uma relação para 2001 entre a média dos valores e a diferença entre os bancos do SIOPS e do FNS para PAB fixo. Esta relação não se apresenta quando se analisa o total das receitas, sugerindo que os municípios de menor porte, ao assumirem a execução de outros programas do MS, passam a se organizar melhor, possibilitando maior qualidade nos dados informados ao SIOPS.

Os achados demonstram, para todas as formas de análises realizadas, uma convergência entre os valores dos bancos de dados analisados para o ano de 2002, sugerindo uma maior qualidade nos dados em relação ao ano anterior. As diferenças encontradas em 2001 para PAB variável são explicadas, provavelmente, devido ao comportamento de variáveis que não foram consideradas neste trabalho por não atenderem aos critérios definidos, como Programa de Combate às Carências Nutricionais, Programa Nacional de Vigilância Epidemiológica e Controle de Doenças, Programa de Assistência Farmacêutica Básica e Ações de Atenção à Saúde dos Povos Indígenas. A única correlação que reduziu em 2002 é para PSF e se deve, provavelmente, ao fato de que nesse ano foi iniciado o pagamento de incentivo ao Programa de Expansão e Consolidação do Programa Saúde da Família (PROESF) e pode não ter sido contabilizado corretamente pelos municípios. Uma comparação dos balancetes municipais com os dados informados ao SIOPS e com os dados registrados pelo FNS permitiria verificar esta hipótese.

As pequenas variações encontradas para MAC no banco do FNS sugerem que os programas de saúde ali agrupados possuíam maior controle e fiscalização, talvez por serem de alto custo e conseqüentemente representarem maiores despesas para a União. Entretanto, as grandes variações e menores correlações observadas neste sistema, quando comparados dois anos, podem significar um ajuste no banco de dados para o ano de 2002. O que não se observa no FINBRA, provavelmente por possuir maior estabilidade nos dados.

Mudanças no SIOPS ocorridas nesse ano podem ter contribuído para o aprimoramento dos dados no sistema: (a) passou a ser integrante do instrumental de controle da Lei de Responsabilidade Fiscal, com periodicidade semestral, possibilitando a geração do demons- trativo da receita líquida de impostos e das despesas próprias com saúde; (b) a utilização dos códigos da Secretaria do Tesouro Nacional para identificar os itens de receita e despesa, facilitando o preenchimento das planilhas; (c) um maior detalhamento de PAB variável, MAC e FAEC, esclarecendo para o usuário do sistema a composição destes itens; (d) a inclusão de planilhas específicas para informações sobre receita e despesa com saneamento; (e) o aprimoramento das críticas internas e da documentação do sistema; (f) uma atuação mais efetiva dos núcleos estaduais, constituídos para apoiar os municípios no preenchimento dos dados e na sua transmissão, realizando atividades de treinamentos em oficinas de trabalho em todo o país.

Da mesma forma, aprimoramentos também ocorreram no sistema do FNS para o ano de 2002, pelo fato de ter desenvolvido um sistema para gerenciamento de transferências fundo a fundo e passado a administrar seu banco de dados apenas em agosto de 2000, o que antes era feito pelo DATASUS. Portanto, os dados relativos ao ano de 2001 são de um sistema bastante novo, ainda em processo de ajustes.

Diferentemente de outros sistemas de âmbito nacional implementados pelo MS, o SIOPS tem em sua estrutura básica uma Câmara Técnica de Orientação e Avaliação, composta por representantes das diversas Secretarias do MS, do Conselho Nacional de Secretários de Saúde (CONASS), do Conselho Nacional de Secretários Municipais de Saúde (CONASEMS), do Conselho Nacional de Saúde (CNS), do Instituto de Pesquisa Econômica e Aplicada (IPEA), do IBGE e do Ministério Público Federal/Procuradoria Federal dos Direitos do Cidadão. Sua missão é, entre outras, propor ações para o aperfeiçoamento dos instrumentos de coleta de dados, mecanismos que assegurem a qualidade das informações e analisar as informações geradas pelo sistema, visando a elaborar indicadores que reflitam a eficácia e a eficiência dos gastos em saúde. A equipe responsável pelo SIOPS, preocupada com a abrangência do sistema e com a qualidade dos dados informados, articulou com a Secretaria do Tesouro Nacional do Ministério da Fazenda o uso de seus dados para avaliação do cumprimento da Lei de Responsabilidade Fiscal e com o Tribunal de Contas da União, para verificação do cumprimento da Emenda Constitucional n. 29.

A partir desses acordos, é inegável a contribuição que o sistema trouxe à ampliação da transparência da gestão e da utilização dos recursos públicos. O sistema amplia as possibilidades de controle e acompanhamento da exe- 
cução da política de saúde por meio de relatórios disponíveis na internet, como os demonstrativos da Lei de Responsabilidade Fiscal e o relatório de despesa liquidada por fonte. Sua disponibilização permite o acesso dos diferentes atores do controle social às informações sobre gastos públicos em saúde, antes restritas a alguns especialistas ou gestores 15,16.

Como o SIOPS coleta informações sobre receita total e despesa com saúde, requer que exista uma interação entre distintas áreas do governo municipal, que nem sempre estão integradas, dificultando a coleta dos dados. Com o uso contínuo do sistema, a rotina de obtenção dos dados se torna mais fácil.

Os dados do SIOPS têm subsidiado o MS na realização de estudos e estimativas sobre gastos públicos nas três esferas de governo, sendo utilizados para estimativas publicadas no Anuário da Saúde 2001 17, nos Indicadores e Dados Básicos em Saúde - IDB 200318 e Cadernos de Informações de Saúde 19. Sendo a mais ampla fonte de dados sobre receita e despesa em saúde no âmbito municipal, seus dados devem ser mais amplamente utilizados, inclusive para estudos que relacionem o financiamento público em saúde com a situação de saúde dos municípios.

\section{Resumo}

Criado em 1999, o Sistema de Informações sobre Orçamentos Públicos em Saúde (SIOPS) coleta informações sobre receita e despesa com saúde das três esferas de governo. A inexistência de outros bancos de dados de abrangência nacional com informações detalhadas sobre gastos municipais faz com que seja a principal fonte de dados para estudos ou estimativas desta natureza. Este trabalho compara os valores de receita declarados pelos municípios ao SIOPS com os registros do Fundo Nacional de Saúde, no intuito de verificar a confiabilidade dos dois bancos de dados e identificar as variáveis com maior discrepância. Compara também os dados de despesa municipal com os registros da Secretaria do Tesouro Nacional. Foi obtido o Coeficiente de Correlação Intraclasses (CCIC) e, em complemento, analisou-se o gráfico de dispersão entre a média dos valores dos dois bancos de dados e a diferença entre estes. Os dados para o ano de 2002 apresentam melhor qualidade. A constatação de que o SIOPS fornece dados confiáveis deve servir de estímulo para que novos estudos considerem o vertente financiamento nas análises de situação de saúde.

Financiamento da Saúde; Sistema de Informação; Sistema de Saúde
Considerando que o FINBRA, em funcionamento desde 1989, portanto estável para o ano de 2001, apresentou diferenças bem inferiores do que as apresentadas na comparação realizada para os dados de receita com o banco de dados do FNS e alta correlação quando comparado com o banco de dados do SIOPS, pode-se inferir que as diferenças encontradas na comparação dos dados de receita sejam consideravelmente influenciadas pelo fato do banco do FNS estar ainda em construção no período analisado.

Este estudo mostra que os dados do SIOPS e do FNS estão bem convergentes para o ano de 2002 e os achados permitem afirmar que os dados disponibilizados são confiáveis para os anos analisados. As informações disponibilizadas pelo sistema, de caráter orçamentário e financeiro, devem ser utilizadas para o planejamento, a gestão e o controle social do financiamento e do gasto público em saúde.

A inclusão dos dados financeiros na composição dos indicadores que explicam a situação de saúde de uma população traz um novo olhar para os estudos epidemiológicos. Os resultados deste trabalho, afirmando que os dados do SIOPS são confiáveis, são um estímulo para que os novos estudos considerem o vertente financiamento na análise de situação de saúde.

\section{Colaboradores}

C. R. A. Lima colaborou na coleta, organização e análise dos dados e elaboração do texto. M. S. Carvalho e J. M. A. Schramm contribuíram na análise dos dados e na elaboração do texto. 


\section{Referências}

1. Brasil. Emenda Constitucional no 29 de 13 de setembro de 2000. Diário Oficial da União 2000; 14 set.

2. Ministério da Saúde/Banco Mundial. A separação do financiamento e da provisão de serviços no Sistema Único de Saúde. Brasília: Ministério da Saúde; 2001.

3. Departamento de Informação e Informática do SUS. Informações financeiras: SIOPS: despesa total com ações e serviços públicos de saúde nas três esferas de governo. http://siops.datasus.gov. br/Documentacao/Despesa_Total_Correntes_PI B_00_03.PDF. (acessado em 25/Mai/2005).

4. Fundo Nacional de Saúde. Gestão financeira do Sistema Único de Saúde: manual básico. 3a Ed. Brasília: Ministério da Saúde; 2003.

5. Medici A. Las reformas de salud em América Latina y Caribe. In: Sanchez H, Zuleta G, organizadores. La hora de los usuarios - reflexiones sobre economía política de las reformas de salud. Washington DC: Banco Interamericano de Desarrollo; 2000. p. 9-26.

6. Carvalho GCM. Financiamento público federal do Sistema Único de Saúde - 1988-2001 [Tese de Doutorado]. São Paulo: Faculdade de Saúde Pública, Universidade de São Paulo; 2002.

7. Departamento de Informação e Informática do SUS. DATASUS: transferência a municípios. http:// portal.saude.gov.br/saude/area.cfm?id_area=347 (acessado em 31/Out/2003).

8. Ministério da Saúde. Fundo Nacional de Saúde: consulta de pagamentos. http://www.fns.saude. gov.br/index.asp (acessado em 31/Out/2003).

9. Piola SF, Biasoto GJ. Finanças e dispêndios: financiamento do SUS, gasto público e base tributária. In: Negri B, Di Giovanni G, organizadores. Brasil. Radiografia da saúde. Campinas: Universidade Estadual de Campinas; 2001. p. 219-32.

10. Ministério da Fazenda. Secretaria do Tesouro Nacional: finanças do Brasil - receita e despesa dos municípios (2001) e (2002). http:/ /www.stn.fazenda.gov.br/estados_municipios/index.asp (acessado em 01/Nov/2004).
11. Departamento de Informação e Informática do SUS. Sistema de informações sobre orçamentos públicos em saúde. http://www.siops.datasus. gov.br (acessado em 31/Out/2003).

12. Bland JM, Altman DG. Statistical methods for assessing agreement between two methods of clinical measurement. Lancet 1986; 1:307-10.

13. Chor D, Coutinho ESF, Laurenti R. Reability of selfreported weight and height among state bank. Rev Saúde Pública 1999; 33:16-23.

14. Landis JR, Koch GG. The measurement of observer agreement for categorical data. Biometrics 1977; 33:159-74.

15. Faveret ACSC. Federalismo fiscal e descentralização no Brasil: o financiamento da política de saúde na década de 90 e início dos anos 2000 [Tese de Doutorado]. Rio de Janeiro: Instituto de Medicina Social, Universidade do Estado do Rio de Janeiro; 2002.

16. Secretaria de Gestão de Investimentos em Saúde. Relatório de atividades 2000. Brasília: Ministério da Saúde; 2001.

17. Departamento de Informação e Informática do SUS. Indicadores de saúde: anuário estatístico de saúde do Brasil. http://portal.saude.gov.br/saude/ aplicacoes/anuario2001/index.cfm (acessado em 01/Nov/2004).

18. Departamento de Informação e Informática do SUS. Indicadores de saúde: indicadores e dados básicos - IDB 2003. http://tabnet.datasus.gov. br/cgi/idb2003/matriz.htm (acessado em 01/Nov/ 2004).

19. Departamento de Informação e Informática do SUS. Indicadores de saúde: caderno de informações de saúde. http://portalweb02.saude.gov. br/ saude/visao.cfm?id_area $=165$ (acessado em 01/ Nov/2004).

Recebido em 13/Abr/2005

Versão final reapresentada em 07/Nov/2005 Aprovado em 06/Dez/2005 